\title{
STABILITY AND OPTIMAL HARVESTING OF A PREY-PREDATOR MODEL WITH STAGE STRUCTURE FOR PREDATOR
}

Abstract. The dynamics of a prey-predator system, where predator has two stages, a juvenile stage and a mature stage, is modelled by a system of three ordinary differential equations. Stability and permanence of the system are discussed. Furthermore, we consider the harvesting of prey species and obtain the maximum sustainable yield and the optimal harvesting policy.

1. Introduction. Stage structured models have received much attention in recent years, i.e., models which take into account the fact that individuals in a population may belong to one of two classes, the immature and mature. See, for example, Aiello and Freedman [1990], Aiello et al. [1992], Freedman and $\mathrm{Wu}$ [1991], Gambell [1985], Landahl and Hanson [1975], Wood et al. [1989] and the references therein. In these models, the age of maturity is represented by a time delay. Cannibalism has been observed in a great variety of species, including a number of fish species. Cannibalism models of various types have also been investigated by Cushing [1991], Diekmann et al. [1986], Freedman et al. [1994], Hastings [1987], Magnusson [1999]. For general models of population growth see Murray [1989]. Recently, papers like Bosch and Gabriel [1997], Kar [2003] and Zhang et al. [2000] study the stage structure of species with or without time delays.

The optimal management of renewable resources, which has a direct relationship to sustainable development, has been studied extensively by many authors. Economic and biological aspects of renewable resources management have been considered by Clark [1976] and other authors like Brauer and Soudack [1979, 1981], Dai and Tang [1998], Kar and Chaudhuri [2000, 2003],

2000 Mathematics Subject Classification: 34C05, 92D25.

Key words and phrases: stage structure, prey-predator, global stability, permanence, maximum sustainable yield, optimal harvesting policy. 
Bhattacharya and Begum [1996], Ragozin and Brown [1985], MestertonGibbons [1988], Leung [1995] and Jerry and Raissi [2001]. From the point of view of ecological managers, it may be desirable for the system to be globally stable or permanent, in order to plan harvesting strategies and keep a sustainable development of the ecosystem.

To facilitate the interpretation of our mathematical findings we assume that the prey or the renewable resource, density of which is denoted by $N_{1}$, can be modelled by a logistic equation when the consumer is absent. We assume that the predators or consumers are divided into two stage groups: juveniles and adults, and their densities are denoted by $N_{2}$ and $N_{3}$ respectively. Here we also assume that only adult predators are capable of preying on the prey species and that the juvenile predators live on their parents. For example, the Chinese alligator can be regarded as a stage-structured species since the mature is more than 10 years old, and can also be regarded as a predator because almost all aquatic animals are the chief food of the Chinese alligator. Another key and somewhat novel feature of our model is to account for the universally prevalent intra-specific competition in the consumer growth dynamics (Kuang et al. [2003]). This intra-specific competition is assumed to induce additional instantaneous deaths only to the adult population and the increased death rate is proportional to the square of the adult population.

With these assumptions, we have the following plausible two-stage preypredator interaction model:

$$
\begin{aligned}
& \frac{d N_{1}}{d t}=r_{1} N_{1}\left(1-\frac{N_{1}}{k}\right)-\alpha N_{1} N_{3}, \quad \frac{d N_{2}}{d t}=\beta N_{3}-r_{2} N_{2}, \\
& \frac{d N_{3}}{d t}=-r_{3} N_{3}+m \alpha N_{1} N_{3}+\gamma N_{2}-\delta N_{3}^{2} .
\end{aligned}
$$

Here $r_{1}$ is the specific growth rate of the prey and $k$ is its carrying capacity. $\alpha$ is the predation parameter; $m$ is the conversion factor; $r_{3}$ is the death rate of mature predator species; $\gamma$ is the proportionality constant of transformation of immature to mature predators; $r_{2}=\mu+\gamma$, where $\mu$ is the death rate; $\beta$ is the birth rate of the immature populations.

Defining

$$
N_{1}=\frac{k r_{2}}{r_{1}} x_{1}, \quad N_{2}=\frac{\beta x_{2}}{m \alpha}, \quad N_{3}=\frac{r_{2} x_{3}}{m \alpha}, \quad t=\frac{\tau}{r_{2}},
$$

we can rewrite (1.1) as

$$
\begin{aligned}
& \frac{d x_{1}}{d \tau}=a x_{1}-x_{1}^{2}-b x_{1} x_{3}, \quad \frac{d x_{2}}{d \tau}=x_{3}-x_{2}, \\
& \frac{d x_{3}}{d \tau}=-c x_{3}+d x_{1} x_{3}+e x_{2}-f x_{3}^{2},
\end{aligned}
$$


where

$$
a=\frac{r_{1}}{r_{2}}, \quad b=\frac{1}{m}, \quad c=\frac{r_{3}}{r_{2}}, \quad d=\frac{m \alpha k}{r_{1}}, \quad e=\frac{\gamma \beta}{r_{2}^{2}}, \quad f=\frac{\delta}{m \alpha} .
$$

In Section 2, we discuss the equilibria and their stability for system (1.2). In Section 3, we consider the harvesting of prey species. Reasonable harvesting policies are indisputably one of the major and interesting problems from the ecological and economical point of view. The exploitation of biological resources and harvests of population species are commonly practised in fishery, forestry and wildlife management. A management of multispecies fisheries is needed to maintain an ecological balance, which is disrupted due to overexploitation of many conventional fish stocks and growing interest in harvesting new kinds of food from the sea. An optimal harvesting policy is studied in Section 4.

2. Equilibria and stability analysis. System (1.2) has to be analyzed with the following initial conditions: $x_{1}(0)>0, x_{2}(0)>0$ and $x_{3}(0)>0$. We observe that the right-hand side of (1.2) is a smooth function of the variables $\left(x_{1}, x_{2}, x_{3}\right)$ and the parameters, as long as these quantities are non-negative, so local existence and uniqueness properties hold in the positive octant. The state space for system (1.2) is the positive octant, $\left\{\left(x_{1}, x_{2}, x_{3}\right): x_{1}>0\right.$, $x_{2}>0$ and $\left.x_{3}>0\right\}$, which is clearly an invariant set, since the vector field on the boundary does not point to the exterior. Our next result concerns the existence of equilibrium points.

We observe that the possible non-negative equilibria of system (1.2) are $P_{0}(0,0,0), P_{1}(a, 0,0)$ and $P_{2}\left(x_{1}^{*}, x_{2}^{*}, x_{3}^{*}\right)$ where

$$
x_{1}^{*}=\frac{a f+b c-b e}{d b+f} \text { and } x_{2}^{*}=x_{3}^{*}=\frac{e+d a-c}{d b+f} .
$$

We remark that if $e>c$, then there exists another equilibrium in the absence of prey. But it is not feasible since prey is the only source of food for the predator. So throughout the paper we assume that $c \geq e$ (i.e., the ratio of the death rate of the mature predators to the rate of transformation from immature to mature is greater than or equal to the ratio of the birth rate and enhanced death rate of the immature predators). Therefore, $P_{2}$ is feasible if $c<e+a d$.

We are particularly interested in the interior equilibrium point $P_{2}\left(x_{1}^{*}\right.$, $\left.x_{2}^{*}, x_{3}^{*}\right)$ for its usual importance.

In order to investigate the stability of system (1.2) near $P_{0}, P_{1}$ and $P_{2}$, we compute the variational matrix which is given by

$$
M\left(x_{1}, x_{2}, x_{3}\right)=\left[\begin{array}{ccc}
a-2 x_{1}-b x_{3} & 0 & -b x_{1} \\
0 & -1 & 1 \\
d x_{3} & e & -c+d x_{1}-2 f x_{3}
\end{array}\right] .
$$


Now,

$$
M(0,0,0)=\left[\begin{array}{ccc}
a & 0 & 0 \\
0 & -1 & 1 \\
0 & e & -c
\end{array}\right]
$$

The characteristic equation of $M(0,0,0)$ is $(a-\lambda)\left\{\lambda^{2}+(c+1) \lambda+c-e\right\}=0$. Hence the point $P_{0}(0,0,0)$ is unstable.

Next,

$$
M(a, 0,0)=\left[\begin{array}{ccc}
-a & 0 & -b a \\
0 & -1 & 1 \\
0 & e & -c+a d
\end{array}\right]
$$

The characteristic equation of $M(a, 0,0)$ is

$$
(a+\lambda)\left\{\lambda^{2}-\lambda(a d-c-1)-(a d+e-c)\right\}=0 .
$$

Therefore $P_{1}(a, 0,0)$ is locally asymptotically stable for $c>e+a d$.

Finally,

$$
M\left(x_{1}^{*}, x_{2}^{*}, x_{3}^{*}\right)=\left[\begin{array}{ccc}
-x_{1}^{*} & 0 & -b x_{1}^{*} \\
0 & -1 & 1 \\
d x_{3}^{*} & e & -c-2 f x_{3}^{*}+d x_{1}^{*}
\end{array}\right]
$$

The characteristic equation of $M\left(x_{1}^{*}, x_{2}^{*}, x_{3}^{*}\right)$ is $\lambda^{3}+A \lambda^{2}+B \lambda+C=0$ where

$$
\begin{aligned}
& A=1+e+x_{1}^{*}+f x_{3}^{*}>0, \\
& B=f x_{3}^{*}+x_{1}^{*}+x_{1}^{*}\left(f x_{3}^{*}+e\right)+b d x_{1}^{*} x_{3}^{*}, \\
& C=(f+b d) x_{1}^{*} x_{3}^{*}>0 .
\end{aligned}
$$

Obviously, $A B-C>0$. According to the Routh-Hurwitz criterion, $P_{2}\left(x_{1}^{*}\right.$, $\left.x_{2}^{*}, x_{3}^{*}\right)$ is locally asymptotically stable if $c<e+a d$.

Now we shall discuss the condition of global stability, permanence and extinction for system (1.2). First, we set

$$
\begin{array}{r}
\mathbb{R}_{+}^{3}=\left\{x=\left(x_{1}, x_{2}, x_{3}\right) \in \mathbb{R}^{3}: x_{i} \geq 0\right\}, \\
\text { Int } \mathbb{R}_{+}^{3}=\left\{x=\left(x_{1}, x_{2}, x_{3}\right) \in \mathbb{R}^{3}: x_{i}>0\right\} .
\end{array}
$$

Definition 2.1. An equilibrium point $P\left(x_{1}, x_{2}, x_{3}\right)$ is said to be globally asymptotically stable in $\mathbb{R}_{+}^{3}$ if it is locally asymptotically stable and all trajectories in $\mathbb{R}_{+}^{3}$ converge to $P\left(x_{1}, x_{2}, x_{3}\right)$.

LEMMA 2.1. (i) If $c \geq e+a d$, then the equilibrium $P_{1}(a, 0,0)$ is globally asymptotically stable in $\mathbb{R}_{+}^{3}$.

(ii) If $c<e+a d$, then the unique interior equilibrium point $P_{2}\left(x_{1}^{*}, x_{2}^{*}, x_{3}^{*}\right)$ is globally asymptotically stable in $\operatorname{Int} \mathbb{R}_{+}^{3}$. 
Proof. (i) We construct the Lyapunov function

$$
V_{1}=\alpha_{1}\left(x_{1}-a-a \ln \frac{x_{1}}{a}\right)+\alpha_{2} x_{2}+\alpha_{3} x_{3}
$$

where $\alpha_{i}, i=1,2,3$, are positive constants to be determined below. Calculating the derivative of $V_{1}$ along each solution of (1.2), we have

$$
\begin{aligned}
\frac{d V_{1}}{d \tau}= & \alpha_{1}\left(\frac{x_{1}-a}{x_{1}}\right) \frac{d x_{1}}{d \tau}+\alpha_{2} \frac{d x_{2}}{d \tau}+\alpha_{3} \frac{d x_{3}}{d \tau} \\
= & -\alpha_{1}\left(x_{1}-a\right)^{2}-\alpha_{1} b\left(x_{1}-a\right) x_{3}+\left(\alpha_{2} x_{3}-c \alpha_{3} x_{3}\right) \\
& -\alpha_{2} x_{2}+\alpha_{3} e x_{2}+\alpha_{3} d x_{1} x_{3}-\alpha_{3} f x_{3}^{2} .
\end{aligned}
$$

Let $\alpha_{1}=d / b, \alpha_{2}=e$ and $\alpha_{3}=1$. Then

$\frac{d V_{1}}{d \tau}=-\frac{d}{b}\left(x_{1}-a\right)^{2}-(c-e-a d) x_{3}-f x_{3}^{2}<0$ in $\operatorname{Int} \mathbb{R}_{+}^{3} \quad$ for $c \geq e+a d$.

Therefore, by Lyapunov-LaSalle (Hale [1969]), it follows that $P_{1}$ is locally asymptotically stable and all trajectories starting in Int $\mathbb{R}_{+}^{3}$ approach $P_{1}$ as $t$ goes to infinity. This establishes the global asymptotic stability.

(ii) Let us take the Lyapunov function

$$
V_{2}\left(x_{1}, x_{2}, x_{3}\right)=\sum \alpha_{i}\left(x_{i}-x_{i}^{*}-x_{i}^{*} \ln \frac{x_{i}}{x_{i}^{*}}\right)
$$

where $\alpha_{i}, i=1,2,3$, are positive constants to be determined below. Calculating the derivative along each solution of (1.2), we have

$$
\begin{aligned}
\frac{d V_{2}}{d \tau}= & \sum \alpha_{i}\left(\frac{x_{i}-x_{i}^{*}}{x_{i}}\right) \frac{d x_{i}}{d \tau} \\
= & -\alpha_{1}\left(x_{1}-x_{1}^{*}\right)^{2}-b \alpha_{1}\left(x_{1}-x_{1}^{*}\right)\left(x_{3}-x_{3}^{*}\right)+\alpha_{2}\left(x_{2}-x_{3}^{*}\right)\left(\frac{x_{3}}{x_{2}}-\frac{x_{3}^{*}}{x_{2}^{*}}\right) \\
& +\alpha_{3} d\left(x_{3}-x_{3}^{*}\right)\left(x_{1}-x_{1}^{*}\right)+e \alpha_{3}\left(x_{3}-x_{3}^{*}\right)\left(\frac{x_{2}}{x_{3}}-\frac{x_{2}^{*}}{x_{3}^{*}}\right) \\
& -f \alpha_{3}\left(x_{3}-x_{3}^{*}\right)^{2} .
\end{aligned}
$$

Let $\alpha_{1}=d / b, \alpha_{2}=e$ and $\alpha_{3}=1$. Then

$$
\begin{aligned}
\frac{d V_{2}}{d \tau}= & -\frac{d}{b}\left(x_{1}-x_{i}^{*}\right)^{2}-f\left(x_{3}-x_{3}^{*}\right)^{2}-e\left(\frac{x_{3}}{x_{2} x_{2}^{*}}\right)\left(x_{2}-x_{2}^{*}\right)^{2} \\
& -e\left(\frac{x_{2}}{x_{3} x_{3}^{*}}\right)\left(x_{3}-x_{3}^{*}\right)^{2}+\frac{2 e}{x_{2}^{*}}\left(x_{2}-x_{2}^{*}\right)\left(x_{3}-x_{3}^{*}\right) \\
= & -\frac{d}{b}\left(x_{1}-x_{i}^{*}\right)^{2}-f\left(x_{3}-x_{3}^{*}\right)^{2} \\
& -e x_{2}^{*}\left[\sqrt{\frac{x_{3}}{x_{2}}}\left(x_{2}-x_{2}^{*}\right)^{2}-\sqrt{\frac{x_{2}}{x_{3}}}\left(x_{3}-x_{3}^{*}\right)\right]^{2}<0 .
\end{aligned}
$$


By similar arguments to those used for $P_{1}$, we conclude that $P_{2}\left(x_{1}^{*}, x_{2}^{*}, x_{3}^{*}\right)$ is globally asymptotically stable if $c<e+a d$.

Definition 2.2. System (1.2) is said to be permanent if there are positive constants $m$ and $M$ such that each positive solution $x\left(t, x_{0}\right)$ of $(1.2)$ with initial condition $x_{0} \in \operatorname{Int} \mathbb{R}_{+}^{3}$ satisfies

$$
m \leq \liminf _{t \rightarrow \infty} x_{i}\left(t, x_{0}\right) \leq \limsup _{t \rightarrow \infty} x_{i}\left(t, x_{0}\right) \leq M, \quad i=1,2,3 .
$$

Definition 2.3. The $i$ th species of system (1.2) is said to be extinctive if each positive solution $x\left(t, x_{0}\right)$ of $(1.2)$ with initial condition $x_{0} \in \operatorname{Int} \mathbb{R}_{+}^{3}$ satisfies

$$
\lim _{t \rightarrow \infty} x_{i}\left(t, x_{0}\right)=0, \quad i=1,2,3 .
$$

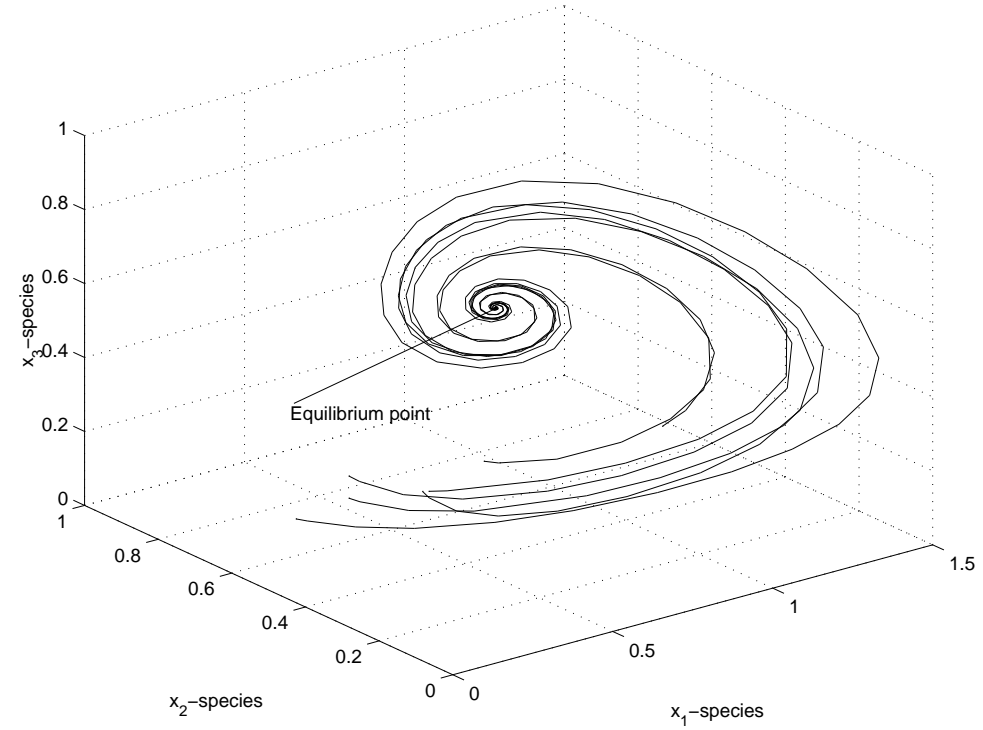

Fig. 1. Phase space trajectories of system (1.2) beginning with different initial levels. It is seen that $P_{2}(0.77,0.56,0.56)$ is a global attractor, where $a=3.0, f=0.04, b=4, c=2.5$, $e=0.2, d=3.0$.

REMARK. We have $c \geq e+a d \Leftrightarrow r_{3} \geq m \alpha k+\gamma \beta / r_{2}$.

We have the following theorem.

THEOREM 2.1. (i) The predator species of system (1.2) is extinctive and the prey species is not extinctive if and only if $c \geq e+a d$.

(ii) Both prey and predator species are permanent if and only if $c<e+a d$.

Proof. This follows easily from Definitions 2.1 and 2.2, and Lemma 2.1.

3. Harvesting of prey species. In order to study the effect of harvesting on system (1.2), let us consider the following system: 


$$
\begin{aligned}
& \frac{d x_{1}}{d \tau}=a x_{1}-x_{1}^{2}-b x_{1} x_{3}-q E x_{1}, \\
& \frac{d x_{2}}{d \tau}=x_{3}-x_{2}, \\
& \frac{d x_{3}}{d \tau}=-c x_{3}+d x_{1} x_{3}+e x_{2}-f x_{3}^{2} .
\end{aligned}
$$

Here $q E x_{1}$ is based on the catch-per-unit-effort hypothesis (Clark [1976]), where $q$ is the catchability coefficient and $E$ is the harvesting effort.

The possible non-negative equilibria of system $(3.1)$ are $Q_{0}(0,0,0)$, $Q_{1}(a-q E, 0,0)$ and $Q_{2}\left(\bar{x}_{1}, \bar{x}_{2}, \bar{x}_{3}\right)$ where

$$
\bar{x}_{1}=\frac{(a-q E) f+b c-b e}{d b+f}, \quad \bar{x}_{2}=\bar{x}_{3}=\frac{e-c+d(a-q E)}{d b+f} .
$$

Similar to the previous section, we have the following theorem.

TheOREM 3.1. (i) The predator species of system (3.1) is extinctive and the prey species is not extinctive if and only if $c+q E d \geq e+a d$.

(ii) System (3.1) is permanent if and only if $c+q E d<e+a d$.

(iii) Both prey and predator species of system (3.1) are extinctive if and only if $a<q E$.

In the following we shall consider the maximum sustainable yield of system (3.1).

Maximum sustainable yield. The maximum sustainable yield (MSY) of a biological resource population is the maximum rate at which it can be harvested even after maintaining the population at a constant level.

Theorem 3.2. The maximum sustainable yield of system (3.1) is equal to

$$
\frac{(a f+b c-b e)^{2}}{4(d b+f) f}
$$

and occurs at the effort level

$$
E_{\mathrm{MSY}}=\frac{a f+b c-b e}{2 q f} .
$$

Proof. Corresponding to a given effort $E$, the sustainable yield is given by

$$
h(E)=q E \bar{x}_{1}=q E \frac{(a-q E) f+b c-b e}{d b+f} .
$$

Then

$$
\frac{d h}{d E}=\frac{q}{d b+f}[(a-q E) f+b c-b e]-\frac{q^{2} E f}{d b+f}
$$


and $d h / d E=0$ gives

$$
\bar{E}=\frac{a f+b c-b e}{2 q f}
$$

Now

$$
\frac{d^{2} h}{d E^{2}}=-\frac{2 q^{2} f}{d b+f}<0 .
$$

Therefore, $h(E)$ is maximum when $E=\bar{E}$. Hence,

$$
\mathrm{MSY}=q \bar{E} \bar{x}_{1}=\frac{(a f+b c-b e)^{2}}{4(d b+f) f} .
$$

Thus the MSY occurs at the effort level $E_{\mathrm{MSY}}=\bar{E}$ and for any value of $E>E_{\mathrm{MSY}}$, the yield $h(E)$ monotonically decreases with $E$ towards zero (see Figure 2). If the effort level exceeds its MSY level, biologists call it a case of biological overexploitation. It is observed that at $E_{\mathrm{MSY}}, Q_{2}$ is globally asymptotically stable.

For simulation let us take $a=3.0, f=0.7, b=4.0, c=0.62, d=3.0$, $e=0.25, q=0.5$. For these values we get $E_{\mathrm{MSY}}=4.95$ and $\mathrm{MSY}=0.38$.

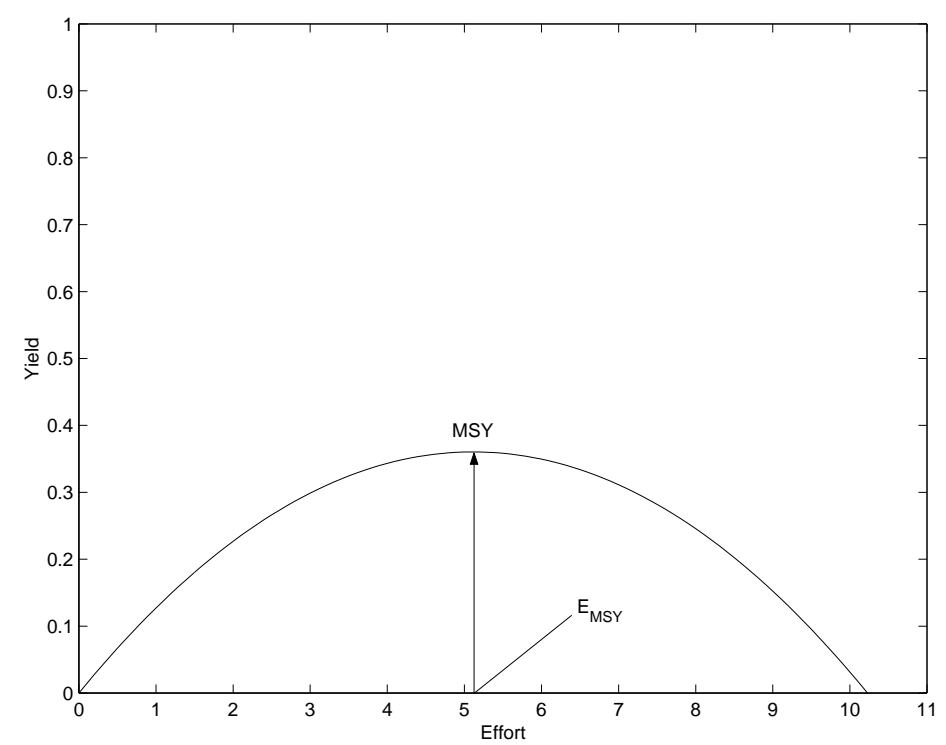

Fig. 2. Yield-effort curve. The curve shows that when $E>E_{\mathrm{MSY}}$, yield monotonically decreases with effort $E$ towards zero.

4. Optimal harvesting policy. The MSY is a simple way to manage resources taking into consideration that overexploiting resources leads to a loss in productivity. Therefore, the aim is to determine how much we can harvest without altering dangerously the harvested population. The main 
problem of the MSY is economical irrelevance. It is so since it takes into consideration the benefits of resource exploitation, but completely disregards its costs. For example, it ignores the fact that if a species is harvested in such a way that its population decreases to a certain level, then the cost of harvesting can become exorbitant because finding the desirable resource becomes more consuming. This might lead to a situation where the cost of harvesting is higher than the benefit. Confronted with the inadequacy of the MSY, people tried to replace it by the "optimal sustainable yield", which is based on the standard cost benefit criterion used to maximize the revenues. Our objective is to solve the following optimization problem:

$$
\max \int_{0}^{\infty} e^{-\theta \tau}\left(p q x_{1}-c\right) E(\tau) d \tau
$$

subject to the state equations of (3.1) and the control constraint $0 \leq E(\tau) \leq$ $E_{\max }$, where $\theta$ is the instantaneous annual discount rate.

In order to solve the above optimization problem, we employ Pontryagin's Maximal Principle (Pontryagin et al. [1964]).

The associated Hamiltonian function is given by

$$
\begin{aligned}
H= & e^{-\theta \tau}\left(p q x_{1}-c\right) E+\lambda_{1}\left[a x_{1}-x_{1}^{2}-b x_{1} x_{3}-q E x_{1}\right]+\lambda_{2}\left[x_{3}-x_{2}\right] \\
& +\lambda_{3}\left[-c x_{3}+d x_{1} x_{3}+e x_{2}-f x_{3}^{2}\right]
\end{aligned}
$$

where $\lambda_{1}, \lambda_{2}$ and $\lambda_{3}$ are adjoint variables and

$$
\phi(\tau)=e^{-\theta \tau}\left(p q x_{1}-c\right)-\lambda_{1} q x_{1}
$$

is called the switching function.

Since $H$ is linear in the control variable, the optimal control will be a combination of the extreme control and the singular control. The optimal control $E(\tau)$ which maximizes $H$ must satisfy the following conditions:

$$
E= \begin{cases}E_{\max } & \text { when } \phi(\tau)>0, \text { i.e. when } \lambda_{1}(\tau) e^{\theta \tau}<p-c / q x_{1}, \\ 0 & \text { when } \phi(\tau)<0, \text { i.e., when } \lambda_{1}(\tau) e^{\theta \tau}>p-c / q x_{1} .\end{cases}
$$

$\lambda_{1}(\tau) e^{\theta \tau}$ is the usual shadow price and $p-c / q x_{1}$ is the net economic revenue on a unit harvest. This shows that $E=E_{\max }$ or zero according as the shadow price is less than or greater than the net economic revenue on a unit harvest. Economically, the first condition implies that if the profit after paying all the expenses is positive, then it is beneficial to harvest up to the limit of available effort. The second condition implies that when the shadow price exceeds the fisherman's net economic revenue on a unit harvest, then the fisherman will not exert any effort.

When $\phi(\tau)=0$, i.e. when the shadow price equals the net economic revenue on a unit harvest, then the Hamiltonian $H$ becomes independent of the control variable $E(\tau)$, i.e., $\partial H / \partial E=0$. This is the necessary and 
sufficient condition for the singular control $E^{*}(\tau)$ to be optimal over the control set $0<E^{*}<E_{\max }$.

Thus the optimal harvest policy is

$$
E(\tau)= \begin{cases}E_{\max }, & \phi(\tau)>0 \\ 0, & \phi(\tau)<0 \\ E^{*}, & \phi(\tau)=0\end{cases}
$$

When $\phi(\tau)=0$, it follows that

$$
\lambda_{1}=e^{-\theta \tau}\left(p q x_{1}-c\right) / q x_{1} .
$$

Now the adjoint equations are

$$
\begin{aligned}
\frac{d \lambda_{1}}{d \tau} & =-\frac{\partial H}{\partial x_{1}}=-\left[e^{-\theta \tau} p q E+\lambda_{1}\left(a-2 x_{1}-b x_{3}-q E\right)+\lambda_{3}\left(d x_{3}\right)\right] \\
\frac{d \lambda_{2}}{d \tau} & =-\frac{\partial H}{\partial x_{2}}=-\left[-\lambda_{2}+\lambda_{3} e\right] \\
\frac{d \lambda_{3}}{d \tau} & =-\frac{\partial H}{\partial x_{3}}=-\left[\lambda_{1}\left(-b x_{1}\right)+\lambda_{2}+\lambda_{3}\left(-c+d x_{1}-2 f x_{3}\right)\right]
\end{aligned}
$$

To obtain an optimal equilibrium solution, we shall use

$$
\left\{\begin{array}{l}
a-x_{1}-b x_{3}-q E=0, \\
x_{3}-x_{2}=0, \\
-c x_{3}+d x_{1} x_{3}+e x_{2}-f x_{3}^{2}=0,
\end{array}\right.
$$

and $x_{1}, x_{2}, x_{3}$ and $E$ can be treated as constants.

By using (4.7), equations (4.4), (4.5) and (4.6) take the form

$$
\left\{\begin{array}{l}
\left(D-x_{1}\right) \lambda_{1}+\left(d x_{3}\right) \lambda_{3}=e^{-\theta \tau} p q E \\
(D-1) \lambda_{2}+e \lambda_{3}=0, \\
\left(D+\left(-c+d x_{1}-2 f x_{3}\right)\right) \lambda_{3}+\lambda_{2}-b x_{1} \lambda_{1}=0,
\end{array}\right.
$$

where $D \equiv d / d \tau$. By eliminating $\lambda_{1}, \lambda_{2}$ from the above differential equations we get

$$
\begin{aligned}
& \left(A_{2} D^{2}+A_{1} D+A_{0}\right) \lambda_{3}=M e^{-\theta \tau}, \\
& A_{2}=1, \\
& A_{1}=-c+d x_{1}-2 f x_{3}-1, \\
& A_{0}=-\left(-c+d x_{1}-2 f x_{3}\right)-e, \\
& M=-\frac{b}{q}\left(p q x_{1}-c\right)(\theta+1) .
\end{aligned}
$$

Therefore, the solution of (4.9) is

$$
\lambda_{3}=B_{1} e^{m_{1} \tau}+B_{2} e^{m_{2} \tau}+\frac{M}{N} e^{-\theta \tau}
$$


where $B_{i}, i=1,2$, are arbitrary constants and the $m_{i}, i=1,2$, are the roots of the characteristic equation of (4.9) and $N=A_{2} \theta^{2}-A_{1} \theta+A_{0} \neq 0$.

It is clear from (4.10) that $\lambda_{3}$ is bounded if and only if $m_{i}<0, i=1,2$, or the $B_{i}, i=1,2$, are identically equal to zero. For robust calculations we ignore the case $m_{i}<0, i=1,2$, and take $B_{i}=0, i=1,2$. Then we have

$$
\lambda_{3}=\frac{M}{N} e^{-\theta \tau} \text {. }
$$

Now substituting $\lambda_{3}$ from (4.11) and $\lambda_{1}$ from (4.3) into the first equation of (4.8) we get the singular path

$$
\frac{p q x_{1}-c}{q x_{1}}\left(\theta+x_{1}\right)-d x_{3} \frac{M}{N}+p\left(a-x_{1}-b x_{3}\right)=0 .
$$

Equation (4.12) together with (4.7) gives the optimal equilibrium population $\left(x_{1}^{*}, x_{2}^{*}, x_{3}^{*}\right)$ and the corresponding optimal harvesting effort $E^{*}$.

5. Concluding remarks. In this paper, we have considered a preypredator model with stage-structure for predator. We have first discussed the existence of possible steady states and then local as well as global stability. An important and interesting question in mathematical ecology is permanence, which ensures the survival of biological species and excludes extinction of species for all positive initial conditions. The question of permanence of biological species is of particular interest to fishery, forestry and wildlife managers. If it is known that a system exhibits such a permanent behaviour, then ecological planning based on a fixed eventual population can be carried out. Analyzing the problem we have obtained conditions for permanence of the solutions of our system.

We have also studied the dynamical behaviour of the system when prey species are subjected to harvesting. Our study indicates a practical danger in the ecological reality. More care should be exercised in setting harvesting rates, since the maximum sustainable yields of the harvesting rates may be far less than what we assumed for the existence of feasible equilibria. Next the optimal harvesting policy is discussed using bang-bang controls and singular controls.

The dynamics exhibited by the system shows good consistence with the observation in biological reality. If the unharvested system is permanent, then a sufficiently small harvesting rate will not change drastically the qualitative behaviour of the system, but the region of coexistence shrinks as the harvesting rate increases. The result provides a theoretical support for safe harvesting in biological resource management.

Before ending this article, we would like to mention that there is still tremendous amount of work to do in this model. For example,

(i) One can consider the stage-structure of prey population.

(ii) The effort level $E$ may be taken as dynamic, i.e. time dependent. 


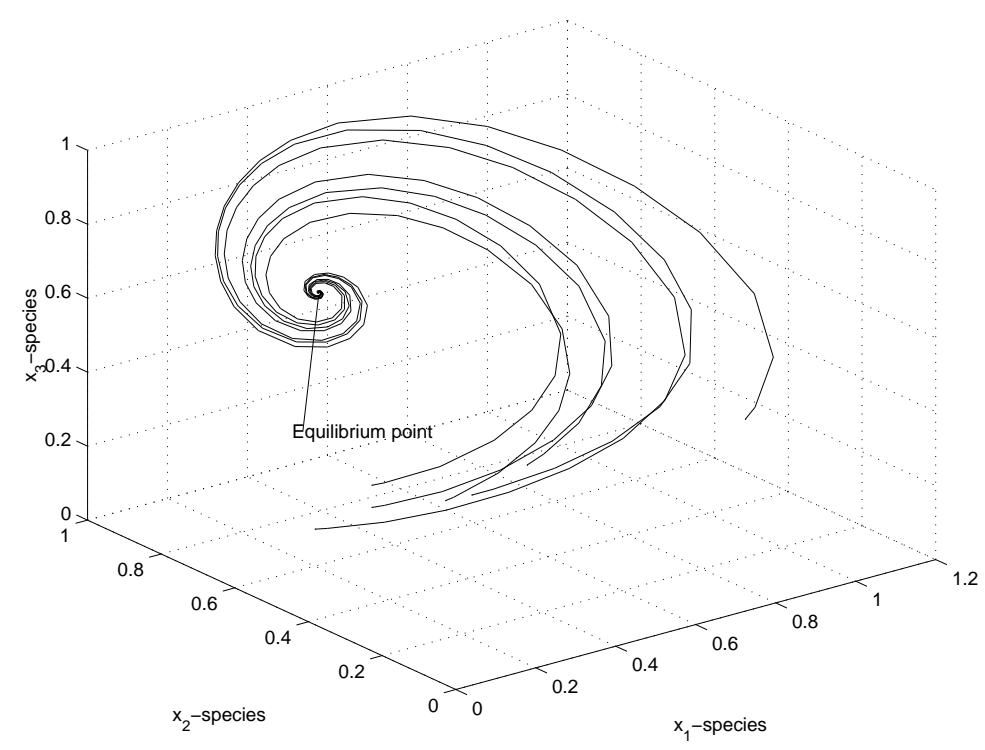

Fig. 3. Phase space trajectories of system (3.1) for $E=E_{\mathrm{MSY}}$. It is seen that the corresponding equilibrium point $(0.16,0.13,0.13)$ is a global attractor.

(iii) Gestation period for predator is also an important characteristic to be considered. We leave it for future considerations.

Acknowledgements. The author would like to thank the referee for valuable suggestions to improve the content of the paper.

\section{References}

W. G. Aiello and H. I. Freedman (1990), A time delay model of single species growth with stage structure, Math. Biosci. 101, 139-150.

W. G. Aiello, H. I. Freedman and J. Wu (1992), Analysis of a model representing stagestructured population growth with state-dependent time delay, SIAM J. Appl. Math. $52,855-869$.

D. K. Bhattacharya and S. Begum (1996), Bionomic equilibrium of two-species system, I, Math. Biosci. 135, 111-127; Erratum, ibid. 151 (1998), 229.

F. van den Bosch and W. Gabriel (1997), Cannibalism in an age-structured predator-prey system, Bull. Math. Biol. 59, 551-567.

F. Brauer and A. C. Soudack (1979), Stability regions and transition phenomena for harvested predator-prey systems, J. Math. Biol. 7, 319-337.

F. Brauer and A. C. Soudack (1981), Coexistence properties of some predator-prey systems under constant rate harvesting, ibid. 12, 101-114.

C. W. Clark 1990, Mathematical Bioeconomics: The Optimal Management of Renewable Resources, 2nd ed., Wiley, New York.

J. M. Cushing, A simple model of cannibalism, Math. Biosci. 107 (1991), 47-71.

G. Dai and M. Tang (1998), Coexistence region and global dynamics of a harvested predatorprey system, SIAM J. Appl. Math. 58, 193-210. 
O. Diekmann, R. M. Nisbet, W. S. C. Gurney and F. van den Bosch (1986), Simple mathematical models for cannibalism: A critique and a new approach, Math. Biosci. $78,21-46$.

H. I. Freedman, J. W.-H. So and J. Wu (1994), A model for the growth of a population exhibiting stage-structure: Cannibalism and cooperation, J. Comput. Appl. Math. 52, 177-198.

H. I. Freedman and J. Wu (1991), Persistence and global asymptotical stability of single species dispersal models with stage structure, Quart. Appl. Math. 49, 351-371.

R. Gambell (1985), Birds and mammals-Antarctic whales, in: Antarctica, W. N. Bonner and D. W. H. Walton (eds.), Pergamon, New York, 223-241.

J. K. Hale (1969), Ordinary Differential Equations, Wiley-Interscience, New York.

A. Hastings (1987), Cycles in cannibalistic egg-larval interactions, J. Math. Biol. 24, 651666.

M. Jerry and N. Raissi (2001), A policy of fisheries management based on continuous fishing effort, J. Biol. Systems 9, 247-254.

T. K. Kar (2003), Selective harvesting in a prey-predator fishery with time delay, Math. Comp. Model. 38, 449-458.

T. K. Kar and K. S. Chaudhuri (2002), On non-selective harvesting of a multi-species fishery, Int. J. Math. Educ. Sci. Technol. 33, 543-556.

T. K. Kar and K. S. Chaudhuri (2003), On non-selective harvesting of two competing fish species in the presence of toxicity, Ecol. Model. 161, 125-137.

Y. Kuang, W. Fagan and I. Loladze (2003), Biodiversity, habitat area, resource growth rate and interference competition, Bull. Math. Biol. 65, 497-518.

H. D. Landahl and B. D. Hanson (1975), A three stage population model with cannibalism, Bull. Math. Biol. 37, 11-17.

A. Leung (1995), Optimal harvesting-coefficient control of steady-state prey-predator diffusive Volterra-Lotka systems, Appl. Math. Optim. 31, 219-241.

K. G. Magnusson (1999), Destabilizing effect of cannibalism on a structured predator-prey system, Math. Biosci. 155, 61-75.

M. Mesterton-Gibbons (1988), On the optimal policy for combined harvesting of independent species, Natural Resource Modelling 2, 107-132.

J. D. Murray (1989), Mathematical Biology, Biomath. 19, Springer.

L. S. Pontryagin, V. S. Boltyanskii, R. V. Gamkrelidze and E. F. Mishchenko (1962), The Mathematical Theory of Optimal Proceses, Wiley-Interscience, New York.

D. L. Ragozin and G. Brown Jr. (1985), Harvest policies and non-market valuation in a predator-prey system, J. Environ. Econ. Management 12, 155-168.

S. N. Wood, S. P. Blythe, W. S. C. Gurney and R. M. Nisbet (1989), Instability in mortality estimation schemes related to stage-structure population models, IMA J. Math. Appl. Med. Biol. 6, 47-68.

X. Zhang, L. Chen and A. U. Neumann (2000), The stage-structure predator-prey model and optimal harvesting policy, Math. Biosci. 168, 201-210.

Department of Mathematics

Bengal Engineering and Science University, Shibpur

Howrah-711 103, India

E-mail: t_k_kar@yahoo.com

Received on 23.3.2004;

revised version on 10.5.2005 